\title{
Norfolk Island
}

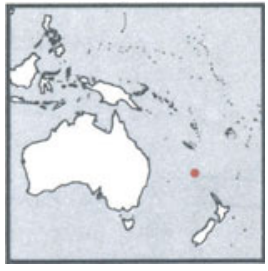

Location South-west Pacific Ocean

The island is in the north Tasman Sea, with

Sydney (Australia) $1500 \mathrm{~km}$ to the south-west

Land Area $36 \mathrm{~km}^{2}=14 \mathrm{mi}^{2}$

Climate Sub-tropical

Weather at Norfolk Island, 99 m altitude

Temperature: hottest month Feb $20-25^{\circ} \mathrm{C}$

coldest Aug $13-18^{\circ} \mathrm{C}$

Rainfall (av monthly): driest month Nov $66 \mathrm{~mm}$, wettest July $155 \mathrm{~mm}$

Time $11 \frac{1}{2}$ hours ahead of GMT

Measures UK (imperial) system, converting to the metric system

Monetary unit Australian dollar $(\mathrm{A} \$)=100$ cents

Rate of exchange (1976 av): free $\$ 1.225=\mathrm{A} \$ 1, \mathrm{~A} \$ 1.474=£ 1$

\section{Summar:}

Political Australian external territory; settled in 1856 by 194 people from Pitcairn Island. There have been proposals for a limited form of self-government. Territorial member of South Pacific Commission Economic There is some agriculture, and forestry is being developed. Tourism is the main foreign exchange earner

\section{People, resources and equipmen!}

Population 1960 790*, $19701560 *, 19761850 *$

Growth: 1960-707.0*\%pa, 1970-762.9*\%pa

Density (1976): $51^{*}$ people per $\mathrm{km}^{2}$

Vital statistics (rate per 1000 people, 1975) : births 10.2, deaths 6.8

Town Kingston (capital)

Race European, Polynesian and European/Polynesian

Language English Labour force (1971) 860

National income per person (1976)

A $\$ 3500 * * *=\$ 4300 * * *=f 2400 * * *$

Telephones (Dec 1976) 400, 220 per 1000 people

Livestock $(000,1976)$ Cattle $2 *$

Roads (1976) $80 * \mathrm{~km}=50 * \mathrm{mi}$, density $2.2 * \mathrm{~km}$ per $\mathrm{km}^{2}$

Port Kingston Airport Norfolk Island

Radio sets (Dec 1975) 1150,615 per 1000 people

\section{Production, finance and trade}

Gross domestic product 1976 est : A $\$ 7 * * * \mathrm{mn}=\$ 8.5 * * * \mathrm{mn}=£ 5 * * * \mathrm{mn}$ Main products Cereals, fruit, vegetables, beef and veal, palm, timber Tourism (1976/77) Number of visitors 18844 Budget (1976/77; year ending June 30th)

Revenue: A $\$ 1.68 \mathrm{mn}=\$ 1.93 \mathrm{mn}=£ 1.13 \mathrm{mn}$

Expenditure: $\mathrm{A} \$ 1.58 \mathrm{mn}=\$ 1.82 \mathrm{mn}=£ 1.07 \mathrm{mn}$

External trade (1976/77; year ending June 30th)

Imports: $A \$ 6.47 \mathrm{mn}=\$ 7.45 \mathrm{mn}=£ 4.35 \mathrm{mn}$

Exports: A $\$ 0.80 \mathrm{mn}=\$ 0.92 \mathrm{mn}=£ 0.54 \mathrm{mn}$

Main imports Food, building materials, petroleum products, radios

Main exports Bean seed, palm seed, fish

Main sources (1974/75) Australia $49 \%$, New Zealand $12 \%$

Main destinations (1974/75) Australia $66 \%$, New Zealand $23 \%$

\section{Pacific Islands, US \\ US Trust Territory of the Pacific Islands and \\ Commonwealth of Northern Marianas}

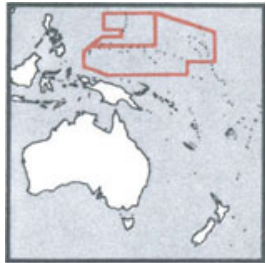

Climate Tropical

Weather at Saipan, Northern Marianas, 206 maltitude

Temperature : hottest month June $24-29^{\circ} \mathrm{C}$, coldest Jan, Feb $22-27^{\circ} \mathrm{C}$

Rainfall (av monthly): driest month Jan $69 \mathrm{~mm}$, wettest Sept $338 \mathrm{~mm}$

Location West Pacific Ocean which about 100 are inhabited; Caroline, Northern Marianas consists of the Marianas excluding Guam. Philippines is to the west and Papua New Guinea to the south

Land Area (land) Northern Marianas

$479 \mathrm{~km}^{2}=185 \mathrm{mi}^{2}$

Trust Territory $1378 \mathrm{~km}^{2}=532 \mathrm{mi}^{\prime}$
Time Northern Marianas and western Caroline Islands (west of $160^{\circ} \mathrm{E}$ ):

10 hours ahead of GMT; Truk island : 11 hours ahead of GMT

Other Caroline islands and Marshall islands: 12 hours ahead of GMT

Measures US system

Monetary unit United States dollar $(\$)=100$ cents

Rate of exchange (1976 av): $\$ 1.806=£ 1$

\section{Summar:}

Political Territory administered by the United States under UN trusteeship since July 18, 1947. In 1975 the Northern Marianas voted to join the United States as a commonwealth, and the former district (Marianas excluding Guam) became a US commonwealth territory from January 1, 1978; figures here in general include those for Northern Marianas. The other trust territories are considering proposals for some form of independence by 1981 when the Trusteeship Agreement ends.

Territorial member of the South Pacific Commission

Economic An agricultural economy with fish and copra the main exports. Tourism is being developed

\section{People, resources and equipment}

Population 1960 74 000*, 197090940,1976123 000*

of which (1976), Northern Marianas 15000*, Trust Territory $108000^{*}$

Growth: 1960-70 2.1*\%pa, 1970-76 5.2*\%pa

Density (1976): Northern Marianas $31^{*}$ people per km²

Trust Territory 78* people per $\mathrm{km}^{2}$

Vital statistics (rate per 1000 people, 1976) : births $28.7^{*}$, deaths $3.0^{*}$

Regions (population in 000, 1973) Northern Marianas 14*

Trust Territory Marshall Islands 25, Caroline Islands 75

(Truk 32, Panape 18, Palau 13, Yap 8, Kosrae 5)

Towns (population in 000, 1973)

Northern Marianas Saipan (capital) 10.8

Trust Territory ${ }^{\mathrm{a}}$ Moen 8.8, Majuro 7.5, Koror 7.2

aAdministered from Saipan

Race Mainly Micronesian and Chamorro; also some Polynesian

Language English, Chamorro, Yapese, Trukese and other Micronesian

Religion (1970) Roman Catholic 50**\%, Protestant 40***\%

Education Pupils (1975/76) 39169 , teachers (1973/74) 1922

Labour force (1970) 14493

Personnel(1975) Physicians: 55, 1 per 2180 people

Standard of living National income per person (1976):

Northern Marianas $\$ 1200 * * *=£ 660 * * *$

Trust Territory $\$ 900 * * *=£ 500 * * *$

Consumption per person (1975): energy $909 \mathrm{~kg}$ coal equivalent,

electricity (production) $960 * \mathrm{~kW} \mathrm{~h}$

Telephones (Dec 1974): 7075,60 per 1000 people

Livestock $(000,1976)$ Cattle $17 *$, pigs $18^{*}$, goats $7 *$, chickens $170 *$

Electrical capacity (1975) 25* megawatts

Hospital beds (1975) 538, 1 per 220 people

Roads (1975) $1035 \mathrm{~km}=643 \mathrm{mi}$, density $0.56 \mathrm{~km}$ per $\mathrm{km}^{2}$

Ports Northern Marianas Saipan, Tinian

Trust Territory Koror, Takatik, Colonia, Moen, Majuro, Kusaie

Airports Northern Marianas Isley (Saipan), Tinian, Rota Carolines Palau,

Ponape, Truk, Yap Marshalls Majuro, Kwajalein

Durable equipment 000 no per

(Dec 1976)

Radio sets

Television sets

Passenger cars 1000 people

$7.2 \quad 67$

no per

$\mathrm{km}$ of road

\section{Production, finance and trade}

Gross domestic product 1976 est: Northern Marianas $\$ 20 * * * \mathrm{mn}=$ $£ 11 * * * \mathrm{mn}$ Trust Territory $\$ 100 * * * \mathrm{mn}=£ 55 * * * \mathrm{mn}$

Main products $(000 \mathrm{t}, 1976)$ Cassava $5^{*}$, sweet potatoes $3 *$,

bananas $2^{*}$, coconuts $84^{*}$, copra $8^{*}$, meat $1^{*}$, fish catch 6.1 ,

electricity (mn kW h, 1975) $115^{*}$

Transport traffic Air (1976) Passenger-km $140 \mathrm{mn}$, cargo 4 mn t-km

Sea (1972) Goods loaded $10000 * \mathrm{t}$, unloaded $80000 * \mathrm{t}$

Budget (1975/76) Revenue: $\$ 72 \mathrm{mn}=£ 38 \mathrm{mn}$

Expenditure: $\$ 62 \mathrm{mn}=£ 33 \mathrm{mn}$

External trade (1976) Imports: $\$ 38^{*} \mathrm{mn}=£ 21 * \mathrm{mn}$

Exports: $\$ 4.8 \mathrm{mn}=£ 2.7 \mathrm{mn}$

Main imports (1976) \% of total Main exports (1976)

Food

Petroleum products

Beverages

Main sources (1972)

United States

$38^{*}$

$20 * *$

$14 *$

Fish

Copra

Handicrafts

Japan
Main destination (1972)

Japan

$27 *$
$\%$ of total

62

54 